\title{
Étalonnage des pratiques de santé publique en matière de gestion de la douleur lors des vaccinations en milieu scolaire
}

\author{
Lucie M Bucci ${ }^{1 \star}$, Noni E MacDonald ${ }^{2,3}$, Tamlyn Freedman ${ }^{4,5}$, Anna Taddio ${ }^{4,5}$
}

\section{Résumé}

Contexte : La douleur et la peur pendant les vaccinations peuvent affecter les enfants et leur comportement futur en rapport avec la vaccination. Ces expériences négatives peuvent être amplifiées lorsque les enfants reçoivent des vaccins dans le cadre de programmes de vaccination à l'école, où le soutien des parents ou des tuteurs fait défaut. En 2015, HELPinKIDS\&ADULTS, un réseau canadien d'experts, a publié un guide de pratiques cliniques sur la gestion de la douleur et de la peur pendant la vaccination. Ce guide a été approuvé par des organisations internationales, nationales et provinciales. Toutefois, le niveau d'intégration et de mise en œuvre du du guide de pratiques cliniques dans les programmes de vaccination locaux et communautaires, tels que les séances de vaccination en milieu scolaire, n'est pas clair.

Méthodes : Une enquête a été effectuer à savoir si les unités de santé publique de l'Ontario avaient intégré et mis en œuvre les interventions contre la douleur et la peur recommandées par le guide de pratiques cliniques dans les politiques et pratiques de vaccination en milieu scolaire.

Résultats : L'étude montre que la majorité des unités de santé publique ont effectivement mis en place des politiques et des procédures relatives à la douleur et à la peur, mais que les interventions ne sont pas intégrées de manière cohérente et formelle, ce qui entraîne une utilisation sous-optimale des interventions lors des vaccinations à l'école.

Conclusion : Pour que les interventions contre la douleur soient appliquées avec suffisamment de fidélité et chez suffisamment de personnes afin d'avoir un effet significatif, les responsables des organisations doivent créer des directives et des procédures qui soutiennent la mise en œuvre de manière systématique et responsable.
Cette oeuvre est mise à la disposition selon les termes de la licence internationale Creative Commons Attribution 4.0

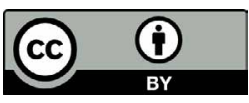

Affiliations

${ }^{1}$ Immunisation Canada, Association canadienne de santé publique, Ottawa, ON

2 Département de pédiatrie, Faculté de médecine, Université Dalhousie, Halifax, NS

${ }^{3}$ Centre de santé IWK, Halifax, NS

${ }^{4}$ Faculté de pharmacie Leslie Dan, Université de Toronto, Toronto, ON

${ }^{5}$ The Hospital for Sick Children, Toronto, ON

\section{${ }^{\star}$ Correspondance :}

Ibucci@cpha.ca

Citation proposée : Bucci LM, MacDonald NE, Freedman T, Taddio A. Étalonnage des pratiques de santé publique en matière de gestion de la douleur lors des vaccinations en milieu scolaire. Relevé des maladies transmissibles au Canada 2020;46(10):413-9. https://doi.org/10.14745/ccdr.v46i10a10f

Mots-clés : immunisation, programmes scolaires, santé publique, douleur, peur, guide de pratiques cliniques, hésitation vaccinale

\section{Introduction}

La vaccination des élèves à l'école est un moyen efficace d'augmenter la couverture vaccinale. Toutes les autorités de santé publique du Canada ont mis en place des programmes scolaires de masse qui permettent de déployer des vaccins contre les méningococcies invasives, I'hépatite $B$ et le virus du papillome humain, entre autres. Les résultats positifs de ces programmes sont nombreux. Par exemple, les vaccins administrés à l'école permettent de mieux suivre les schémas posologiques multiples et de vacciner au bon moment par rapport au moment où la protection est nécessaire (1). En outre, les vaccins faits à temps réduisent le risque d'infection et les effets négatifs sur la santé.

Un nombre important d'élèves qui se font vacciner à l'école ont des expériences négatives; ces réactions peuvent conduire à un refus futur de se faire vacciner (2). Ces expériences négatives sont souvent liées à la douleur et à la peur des aiguilles.

Deux personnes sur trois ont peur de la douleur causée par les aiguilles (3). Les élèves qui éprouvent de la peur peuvent avoir des épisodes d'évanouissement, des maux de tête, des 
nausées et d'autres symptômes. Ces réactions liées au stress de la vaccination peuvent se produire en grappes et, si elles ne sont pas bien gérées, peuvent saper la confiance dans les programmes de vaccination (4).

Il existe des interventions fondées sur des données probantes pour atténuer les expériences négatives dues à la douleur et à la peur lors des vaccinations. En 2015, HELPinKIDS\&ADULTS, un réseau canadien d'experts, a publié un guide de pratiques cliniques sur la gestion de la douleur et de la peur pendant la vaccination (5). L'Organisation mondiale de la Santé (OMS) a reconnu ce guide et y a fait référence dans son premier document d'orientation mondial sur la réduction de la douleur au moment de la vaccination (6). Plus récemment, l'Agence de la santé publique du Canada a intégré ce guide dans le Guide canadien d'immunisation (7). En outre, le ministère de la Santé et des Soins de longue durée de l'Ontario (MSSLD) et le Centre de contrôle des maladies de la Colombie-Britannique (BCCDC) ont tous deux adopté le guide dans leurs politiques et procédures de vaccination $(8,9)$.

Bien que le guide ait été approuvé aux niveaux international, national et provincial, on ne sait pas exactement combien d'unités de santé publique ont intégré et mis en œuvre les recommandations du guide de pratiques cliniques dans leurs programmes de vaccination en milieu scolaire.

Étant donné que les programmes de vaccination scolaire sont menés au niveau local, il est important d'étudier ce que font les unités locales de santé publique pour intégrer et mettre en œuvre pleinement le guide. II ne fait guère de doute qu'il faut du temps pour apporter de vastes changements dans les pratiques de santé publique. La volonté de changement peut également ne pas être présente aux niveaux local et/ou communautaire.

En 2015, le MSSLD a publié une feuille de route pour améliorer I'immunisation dans la province. Ce plan a défini le guide de pratiques cliniques 2015 comme une approche fondée sur des données probantes pour améliorer l'expérience en matière de vaccination, renforcer l'administration des vaccins et réduire les obstacles à la vaccination (10).

Pour comprendre l'état d'avancement de la mise en œuvre du guide de pratiques cliniques, nous avons entrepris une étude comparative afin de documenter les pratiques de gestion de la douleur et les obstacles et facilitateurs de l'adoption du guide de pratiques cliniques par les unités de santé publique de l'Ontario.

\section{Méthodes}

Pendant la période couverte par notre enquête (de septembre 2017 à mars 2019), le programme de vaccination en milieu scolaire en Ontario a permis d'administrer trois vaccins aux élèves de septième année : une dose du vaccin antiméningococcique et deux doses des vaccins contre l'hépatite $B$ et le virus du papillome humain. Un membre de l'équipe de recherche d'Immunisation Canada a envoyé par courriel une invitation à un contact dans chaque unité de santé publique afin d'identifier un membre du personnel connaissant les politiques et les pratiques de vaccination en milieu scolaire et qualifié pour répondre à l'enquête. Le contact a été identifié à partir d'une liste tenue par Immunisation Canada et vérifiée par le MSSLD. Au moyen d'un entretien téléphonique structuré, un enquêteur formé a mené l'enquête et a interrogé le membre du personnel identifié de chaque unité (i.e. une infirmière de santé publique, un responsable des maladies évitables par la vaccination, un superviseur ou un directeur de séances de vaccination).

Le guide d'entretien a été conçu selon les directives standard de conception des enquêtes. Le guide comprenait 53 questions en format binaire, à choix multiples et ouvertes. Les questions portaient sur les domaines suivants : la présence de politiques concernant les vaccinations scolaires, le processus de consentement, la formation du personnel et des élèves sur la manière de faire face à la douleur et à la peur pendant l'injection, les interventions utilisées lors des séances de vaccination, l'évaluation des symptômes (douleur, peur) et de la satisfaction des élèves, les obstacles perçus à l'atténuation de la douleur, et les caractéristiques de l'unité (i.e. taille de la population desservie). L'enquête durait environ 45 à 60 minutes.

L'enquête a été pré-testée auprès des membres de la Coalition canadienne des infirmières et infirmiers pour l'immunisation (CCIII). Cette coalition nationale d'infirmières du secteur de la santé publique et des ministères provinciaux de la Santé connaît bien la politique et les procédures des programmes de vaccination scolaire.

L'approbation éthique a été obtenue auprès du comité d'éthique de la recherche du Centre de santé IWK

L'approbation éthique a été obtenue auprès du comité d'éthique de la recherche du Centre de santé IWK.

\section{Résultats}

Sur les 36 unités de santé publique éligibles, des personnes de 24 unités ont participé à l'enquête. Six répondants (25\%) provenaient d'unités de santé publique desservant une population de 500000 personnes ou plus.

Les réponses des différents domaines d'enquête sont résumées dans le tableau 1. Tous les répondants ont déclaré que leur unité de santé publique avait une politique locale pour superviser la planification, la mise en œuvre et la communication des pratiques de vaccination dans les écoles. En outre, 14 personnes sur 24 (58\%) ont déclaré que leur unité avait mis en place des politiques de gestion de la douleur et de la 
peur. La majorité des répondants $(n=19 ; 79 \%)$ ont indiqué qu'ils éduquent officiellement les élèves avant les séances de vaccination scolaires. Cette éducation comprend généralement la justification de la vaccination, le fonctionnement des vaccins et leurs effets secondaires potentiels. Aucune des personnes interrogées n'a déclaré avoir fourni des informations aux élèves sur la manière de faire face à la douleur ou à la peur des aiguilles.

\section{Tableau 1 : Caractéristiques des programmes de vaccination scolaire dans les unités de santé publique de l'Ontario $(n=24)$}

\begin{tabular}{|l|r|}
\multicolumn{1}{|c|}{ Sujet de l'enquête } & \multicolumn{2}{c|}{$\begin{array}{c}\text { Nombre de } \\
\text { personnes ayant } \\
\text { répondu « oui » à } \\
\text { la question }\end{array}$} \\
\hline Ont des politiques de vaccination à l'école portant sur : \\
\hline Le processus d'administration des vaccins & 24 \\
\hline La gestion de l'anaphylaxie & 24 \\
\hline Les procédures des séances de vaccination & 24 \\
\hline La gestion des évanouissements & 22 \\
\hline $\begin{array}{l}\text { L'éducation et la communication avec les } \\
\text { élèves }\end{array}$ & 19 \\
\hline $\begin{array}{l}\text { L'éducation et la communication avec le } \\
\text { personnel des écoles }\end{array}$ & 18 \\
\hline La gestion de la douleur et de la peur & 14 \\
\hline L'évaluation/la qualité du programme & 12 \\
\hline Ont des procédures de consentement & 20 \\
\hline Envoyées au domicile avec les élèves & 1 \\
\hline Envoyées par courrier aux parents & 1 \\
\hline $\begin{array}{l}\text { Acceptation du consentement des élèves } \\
\text { du secondaire }\end{array}$ & 2 \\
\hline Autres : non expliqué & \\
\hline$E$ éd & \\
\hline
\end{tabular}

Éduquent les élèves avant la séance de vaccination sur ${ }^{\mathrm{b}}$ :

La raison du vaccin, son mode d'action et ses effets secondaires

La raison du vaccin, son mode d'action, ses

effets secondaires et les exemptions

La raison du vaccin, son mode d'action, ses effets secondaires, la logistique de la journée du vaccin et le site web pour la foire aux questions

La raison du vaccin, son mode d'action, ses effets secondaires, ses risques et ses avantages

La raison du vaccin et ses effets secondaires

Dispensent une formation officielle au personnel de santé publique sur :

Le traitement de l'anaphylaxie ${ }^{a}$

L'atténuation des évanouissements

$L^{\prime}$ administration du programme

La communication avec le personnel des écoles ${ }^{\mathrm{a}}$

La communication avec les élèves ${ }^{a}$

\section{Tableau 1 : Caractéristiques des programmes de vaccination scolaire dans les unités de santé publique de l'Ontario $(n=24)$ (suite)}

\begin{tabular}{|l|c|} 
Sujet de l'enquête & $\begin{array}{c}\text { Nombre de } \\
\text { personnes ayant } \\
\text { répondu « oui » à } \\
\text { la question }\end{array}$ \\
\hline L'atténuation de la douleur $^{\mathrm{a}}$ & 12
\end{tabular}

\begin{tabular}{|l|r}
\hline L'atténuation de la peur et de l'anxiété & 12 \\
\hline
\end{tabular}

Surveillent les élèves pendant les séances de vaccination pour déterminer :

Les préférences des élèves en matière de stratégies d'adaptation ${ }^{\mathrm{a}}$

Documentées dans une base de données informatique

Documentées manuellement

L'expérience des élèves en matière de vaccination $^{\mathrm{a}}$

Aucune trace écrite de la manière dont c'est indiqué

La peu

La peur

Description informelle (non

documentée) de la manière dont c'est indiqué

La douleur ${ }^{\mathrm{a}}$

Description informelle (non

documentée) de la manière dont c'est indiqué

Base de données informatique

Pas de réponse

a $\mathrm{n}=1$ manquant

$n=2$ manquants

Les interventions d'adaptation ont été appliquées de manière incohérente au cours des séances de vaccination (tableau 2). Alors que la majorité des répondants ont déclaré avoir utilisé diverses interventions d'adaptation, moins de la moitié ont déclaré avoir «toujours» ou «presque toujours» utilisé des interventions d'adaptation, à l'exception de la distraction verbale, des sièges confortables, des temps d'attente courts et de la vaccination prioritaire des élèves les plus craintifs. Peu de répondants ont sollicité des élèves les préférences en matière d'adaptation, l'expérience globale de la vaccination ou les symptômes liés à l'injection (douleur, peur).

Les répondants ont identifié plusieurs défis qui ont affecté la mise en œuvre des interventions visant à atténuer la douleur et la peur. II s'agissait notamment de contraintes budgétaires et de contraintes d'espace physique dans les écoles. En outre, les personnes interrogées ont déclaré être incapables de négocier des espaces optimaux pour les séances de vaccination dans les écoles en raison de mauvaises relations avec les administrateurs scolaires. 
Tableau 2 : Interventions contre la douleur et la peur appliquées par les unités de santé publique en Ontario $(n=24)$

\begin{tabular}{|c|c|c|c|}
\hline Intervention & $\begin{array}{l}\text { Nombre de } \\
\text { ceux qui ont } \\
\text { recours à } \\
\text { l'intervention }\end{array}$ & \multicolumn{2}{|c|}{$\begin{array}{l}\text { Fréquence } \\
\text { d'utilisation de } \\
\text { l'intervention }\end{array}$} \\
\hline \multirow{6}{*}{$\begin{array}{l}\text { Distraction avec des } \\
\text { objets personnels } \\
\text { Les articles personnels } \\
\text { utilisés comprennent } \\
\text { le téléphone portable } \\
\text { personnel }(n=21) \text { et/ou } \\
\text { un autre objet }(n=1)\end{array}$} & \multirow{6}{*}{22} & Toujours & 6 \\
\hline & & $\begin{array}{l}\text { Presque } \\
\text { toujours }\end{array}$ & 4 \\
\hline & & Parfois & 9 \\
\hline & & Presque jamais & 1 \\
\hline & & Jamais & 0 \\
\hline & & Pas de réponse & 2 \\
\hline \multirow{6}{*}{ Distraction verbale ${ }^{a}$} & \multirow{6}{*}{23} & Toujours & 8 \\
\hline & & $\begin{array}{l}\text { Presque } \\
\text { toujours }\end{array}$ & 5 \\
\hline & & Parfois & 3 \\
\hline & & Presque jamais & 0 \\
\hline & & Jamais & 0 \\
\hline & & Pas de réponse & 5 \\
\hline \multirow{6}{*}{$\begin{array}{l}\text { S'asseoir dans une } \\
\text { position confortable }\end{array}$} & \multirow{6}{*}{20} & Toujours & 11 \\
\hline & & $\begin{array}{l}\text { Presque } \\
\text { toujours }\end{array}$ & 2 \\
\hline & & Parfois & 0 \\
\hline & & Presque jamais & 1 \\
\hline & & Jamais & 0 \\
\hline & & Pas de réponse & 6 \\
\hline \multirow{6}{*}{ Délais d'attente court ${ }^{\mathrm{a}}$} & \multirow{6}{*}{18} & Toujours & 10 \\
\hline & & $\begin{array}{l}\text { Presque } \\
\text { toujours }\end{array}$ & 5 \\
\hline & & Parfois & 3 \\
\hline & & Presque jamais & 0 \\
\hline & & Jamais & 0 \\
\hline & & Pas de réponse & 0 \\
\hline \multirow{6}{*}{$\begin{array}{l}\text { Présence du soutien } \\
d^{\prime} u n \text { pairb }\end{array}$} & \multirow{6}{*}{18} & Toujours & 0 \\
\hline & & $\begin{array}{l}\text { Presque } \\
\text { toujours }\end{array}$ & 6 \\
\hline & & Parfois & 8 \\
\hline & & Presque jamais & 1 \\
\hline & & Jamais & 0 \\
\hline & & Pas de réponse & 3 \\
\hline
\end{tabular}

Tableau 2 : Interventions contre la douleur et la peur appliquées par les unités de santé publique en Ontario ( $n=24)$ (suite)

\begin{tabular}{|l|l} 
Intervention & $\begin{array}{c}\text { Nombre de } \\
\text { ceux qui ont } \\
\text { recours à } \\
\text { I'intervention }\end{array}$
\end{tabular}

\section{Intimitéc}

Les approches utilisées pour assurer l'intimité dépendent de l'école $(n=6)$ et comprennent I'utilisation d'une salle privée $(n=5)$, la vaccination des élèves au début ou à la fin de la séance $(n=3)$ et l'utilisation d'une cloison d'intimité $(n=1)$ d'anesthésiques topiques $^{\mathrm{a}}$

Utilisation
d'anesthésiques $^{\text {topiques }}$

Vacciner d'abord les plus craintifs ${ }^{a}$

\begin{tabular}{|c|c|c|c|}
\hline & & \\
\hline & & Jamais & 0 \\
\hline & & Pas de réponse & 3 \\
\hline \multirow{6}{*}{$\begin{array}{l}\text { Vacciner d'abord les } \\
\text { plus craintifs }{ }^{a}\end{array}$} & \multirow{6}{*}{15} & Toujours & 8 \\
\hline & & $\begin{array}{l}\text { Presque } \\
\text { toujours }\end{array}$ & 3 \\
\hline & & Parfois & 2 \\
\hline & & Presque jamais & 1 \\
\hline & & Jamais & 0 \\
\hline & & Pas de réponse & 1 \\
\hline $\begin{array}{l}\text { Distraction assurée par } \\
\text { la santé publique }\end{array}$ & \multirow{6}{*}{12} & Toujours & 2 \\
\hline \multirow{5}{*}{$\begin{array}{l}\text { Parmi les articles } \\
\text { utilisés, citons les jus } \\
\text { de fruits, les balles } \\
\text { antistress, les jouets } \\
\text { (e.g. les bulles, les roues } \\
\text { à aubes, les peluches), } \\
\text { les livres }\end{array}$} & & $\begin{array}{l}\text { Presque } \\
\text { toujours }\end{array}$ & 1 \\
\hline & & Parfois & 4 \\
\hline & & Presque jamais & 1 \\
\hline & & Jamais & 0 \\
\hline & & Pas de réponse & 4 \\
\hline
\end{tabular}

$\mathrm{n}=1$ manquant

b $\mathrm{n}=3$ manquants

${ }^{c} \mathrm{n}=2$ manquants

\section{Discussion}

La mise en œuvre d'interventions dont il est prouvé qu'elles soulagent la douleur et la peur lors des séances de vaccination en milieu scolaire nécessite l'intégration de toutes les politiques et pratiques de vaccination et la collaboration de toutes les parties prenantes (santé publique, personnel scolaire, élèves, parents). À l'heure actuelle, les recommandations du guide de pratiques cliniques semblent être insuffisamment intégrées dans les activités de planification et de prestation des séances 
de vaccination. Cela limite le personnel de première ligne dans sa capacité à mettre en œuvre différents types d'interventions contre la douleur à la fréquence requise.

Pour parvenir à une utilisation plus large et plus cohérente des interventions, nous recommandons aux responsables de la santé publique et aux gestionnaires de politiques et de programmes d'élaborer des politiques et des procédures qui intègrent explicitement les interventions visant à atténuer la douleur dans les activités de planification et de prestation des séances de vaccination et qui suivent les symptômes des élèves comme indicateur de qualité de leurs programmes.

Un cadre d'administration des vaccins, le système CARDTM, a été mis au point pour faciliter l'intégration du guide de pratiques cliniques dans le programme de vaccination scolaire (11). Chaque lettre du mot «CARD» englobe une catégorie d'interventions fondées sur des preuves ( $\mathrm{C}$ - Confort, A - Aide, R - Relaxation, $D$ - Distraction) pour réduire la douleur, la peur et les évanouissements. Toutes ces interventions peuvent être utilisées à l'avance pour préparer les élèves à la vaccination.

Le système CARD TM propose une approche systématique de la planification, de la séance de vaccination et du suivi des vaccinations en milieu scolaire. Le système tient compte des besoins et des préférences des élèves, en favorisant les soins axés sur l'élève et l'équité en matière de santé (12). Le programme prévoit, par exemple, de rencontrer à l'avance les directeurs d'école pour identifier les espaces appropriés à utiliser pour la séance de vaccination, de préparer les espaces afin de minimiser les éléments qui suscitent la peur et de demander aux élèves de signaler leurs symptômes.

Pour réussir, CARDTM nécessite le soutien des responsables organisationnels qui créent des politiques et des procédures pour soutenir la mise en œuvre. L'éducation et la formation de tous les acteurs concernés sont également nécessaires afin qu'ils connaissent le programme, s'y engagent et puissent le mettre en œuvre avec fidélité (13).

À ce jour, CARDTM a été évalué dans une unité de santé publique en Ontario, le service de santé publique de la région de Niagara, où il a prouvé qu'il réduisait la peur et les étourdissements des élèves (un précurseur de l'évanouissement) lors des vaccinations en milieu scolaire (14). Après son évaluation, CARDTM a été intégré à l'ensemble du programme de vaccination scolaire de l'unité.

Il y a plusieurs avantages potentiels à traiter la douleur et la peur lors des vaccinations scolaires. Tout d'abord, les élèves auront une expérience plus positive de la vaccination, car ils auront moins de douleur et de peur. Deuxièmement, l'utilisation de stratégies d'atténuation de la douleur et de la peur permet aux élèves d'acquérir des compétences d'adaptation qui peuvent être appliquées à d'autres situations stressantes. Troisièmement, en réduisant la douleur et la peur, les infirmières font preuve à la fois de compétence et d'attention, qualités nécessaires pour développer des relations de confiance. À long terme, les élèves peuvent développer des attitudes plus positives à l'égard de la vaccination et des fournisseurs de soins de santé, ce qui peut conduire à une meilleure acceptation des vaccins et d'autres interventions de soins de santé, et donc à de meilleurs résultats pour la santé (15).

\section{Limites}

Il convient de reconnaître quelques lacunes, notamment les changements de pratiques au fil du temps, les erreurs potentielles de mesure et les erreurs potentielles d'interprétation des données. L'étude s'est déroulée entre 2017 et 2019, et il est possible que certaines pratiques des unités de santé publique aient changé après avoir participé à cette étude. Par exemple, comme indiqué précédemment, une unité de santé publique qui a participé au développement de CARD TM I'a ensuite intégré dans l'ensemble du programme scolaire en 2019.

En outre, les participants ont fait part de leurs propres pratiques et peuvent avoir introduit des erreurs aléatoires et des préjugés (i.e. un préjugé de désirabilité sociale) dans leurs réponses. Toutefois, vu que les résultats ont démontré une faible adoption des recommandations du guide de pratiques cliniques en général, il est peu probable que les préjugés aient contribué à une source d'erreurs importantes.

Enfin, la conception de l'étude ne permet pas d'estimer les taux d'utilisation réels des interventions spécifiques contre la douleur, et les réponses peuvent être interprétées par inadvertance comme une utilisation réelle. Par exemple, alors que la plupart des répondants ont indiqué qu'ils utilisent presque toujours ou toujours des articles de distraction personnelle, cette intervention exige que les élèves viennent à l'école avec ces articles en main. Étant donné que les élèves et leurs familles ne sont pas informés à l'avance de la possibilité d'utiliser des dispositifs personnels de distraction pendant les séances de vaccination, il est probable que beaucoup d'entre eux n'apportent rien pour se distraire.

\section{Conclusion}

Cette enquête menée auprès des unités de santé publique de l'Ontario a montré que l'intégration et la mise en œuvre du guide de vaccination contre la douleur dans la pratique des séances scolaires au niveau local étaient limitées. Bien que la majorité des unités de santé publique disposent de politiques et de procédures sur la gestion de la douleur et de la peur, elles ne semblent pas être mises en œuvre de manière formelle par le personnel de santé publique. Cela conduit à une intégration incohérente et sous-optimale des interventions contre la douleur dans le programme de vaccination scolaire. Pour que les interventions contre la douleur soient appliquées avec suffisamment de fidélité et chez suffisamment de personnes pour avoir un effet significatif, les responsables des organisations 
doivent créer des directives et des procédures qui soutiennent la mise en œuvre de manière systématique et responsable.

\section{Déclaration des auteurs}

L. M. B. - Administration du projet, supervision, enquête, conservation des données, analyse formelle, rédaction originale N. E. M. - Conceptualisation, rédaction-révision et édition T. F. - Conservation des données, rédaction-révision et édition A. T. - Conceptualisation, méthodologie, supervision, rédaction-révision et édition

\section{Intérêts concurrents}

Cette étude a été réalisée par l'Association canadienne de santé publique/Immunisation Canada avec des collaborateurs de I'Université de Toronto et de l'Université Dalhousie. II n'y a pas d'autres divulgations.

\section{Remerciements}

Les auteurs tiennent à remercier les personnes qui ont participé à cette étude, l'équipe HELPinKIDS\&ADULTS, ainsi que L. Lee et R. Segall pour leur contribution.

\section{Financement}

A. T. rapporte la section 9 de la marque $n^{\circ} 924835$ pour CARD.

L. M. B. rapporte qu'Immunisation Canada a reçu des subventions de Pfizer Canada, Merck Canada, GSK Canada, Seqirus Canada et Sanofi Pasteur en dehors des travaux présentés.

\section{Références}

1. Perman S, Turner S, Ramsay Al, Baim-Lance A, Utley M, Fulop NJ. School-based vaccination programmes: a systematic review of the evidence on organisation and delivery in high income countries. BMC Public Health 2017 Mar;17(1):252-64. DOI PubMed

2. Taddio A, Chambers CT, Halperin SA, Ipp M, Lockett D, Rieder MJ, Shah V. Inadequate pain management during routine childhood immunizations: the nerve of it. Clin Ther 2009;31 Suppl 2:S152-67. DOI PubMed

3. Taddio A, Ipp M, Thivakaran S, Jamal A, Parikh C, Smart S, Sovran J, Stephens D, Katz J. Survey of the prevalence of immunization non-compliance due to needle fears in children and adults. Vaccine 2012 Jul;30(32):4807-12. DOI PubMed
4. Gold MS, MacDonald NE, McMurtry CM, Balakrishnan MR, Heininger U, Menning L, Benes O, Pless R, Zuber PL. Immunization stress-related response - Redefining immunization anxiety-related reaction as an adverse event following immunization. Vaccine 2020 Mar;38(14):3015-20. DOI PubMed

5. Taddio A, McMurtry CM, Shah V, Riddell RP, Chambers CT, Noel M, MacDonald NE, Rogers J, Bucci LM, Mousmanis $P$, Lang E, Halperin SA, Bowles S, Halpert C, Ipp M, Asmundson GJ, Rieder MJ, Robson K, Uleryk E, Antony MM, Dubey V, Hanrahan A, Lockett D, Scott J, Bleeker EV; HELPinKids\&Adults. Reducing pain during vaccine injections: clinical practice guideline. CMAJ 2015 Sep;187(13):975-82. DOI PubMed

6. Organisation mondiale de la Santé. Réunion du Groupe stratégique consultatif d'experts sur la vaccination, avril 2015 : conclusions et recommandations [REH]. Relevé épidémiologique hebdomadaire 2015 mai;90(22):261-78. https://www.who.int/wer/2015/wer9022.pdf?ua=1

7. Agence de la santé publique du Canada. Méthodes d'administration des vaccins : Guide canadien d'immunisation. Tableau 4 : Stratégies de gestion de la douleur associée à la vaccination, en fonction des groupes d'âge. Ottawa (ON) : Agence de la santé publique du Canada (modifié 2017-11-03; accédé 2020-07-13). https://www.canada.ca/fr/sante-publique/ services/publications/vie-saine/guide-canadie n-immunisation-partie-1-information-cle-immunisation/page8-methodes-administration-vaccins.html

8. Ministère de la Santé et des Soins de longue durée de I'Ontario. Les vaccins : Remède par excellence : Rapport annuel 2014 du médecin-hygiéniste en Chef de l'Ontario : Imprimeur de la Reine pour l'Ontario, 2016. Catalogue Numéro de catalogue : 020370 ISSN 1920-9312. http:// www.health.gov.on.ca/fr/common/ministry/publications/ reports/cmoh_14_vaccines/docs/cmoh_14_vaccines.pdf

9. BC Centre for Disease Control. Communicable Disease Control Manual, Chapter 2: Immunization. Appendix D - reducing immunization injection pain. Vancouver $(B C)$ : BCCDC; 2018-12. http://www.bccdc.ca/resource-gallery/ Documents/Guidelines\%20and\%20Forms/Guidelines\%20and\%20 Manuals/Epid/CD\%20Manual/Chapter\%202\%20-\%20lmms/ SectionIVB_RIIP.pdf

10. Ministère de la Santé et des Soins de longue durée de I'Ontario. Rapports du ministère : Immunisation 2020 : Modernisation du programme de vaccination financé par le secteur public en Ontario. Toronto (Ontario) : MSMSLD; 2015-12 (accédé 2020-07-15). http://www.health. gov.on.ca/en/common/ministry/publications/reports/ immunization_2020/

11. Taddio A. Pain Pain Go Away: improving the vaccination experience at school. Paediatr Child Health 2019 Apr;24 Suppl 1:S1-2. DOI PubMed 
12. Taddio A, McMurtry CM, Bucci LM, MacDonald N, Ilersich AN, Ilersich AL, Alfieri-Maiolo A, deVlaming-Kot C, Alderman L; Pain Pain Go Away Team. Overview of a Knowledge Translation (KT) Project to improve the vaccination experience at school: the CARD ${ }^{\mathrm{TM}}$ System. Paediatr Child Health 2019 Apr;24 Suppl 1:S3-18. DOI PubMed

13. Taddio A, Alderman L, Freedman T, McDowall $T$, McMurtry CM, MacDonald N, deVlaming-Kot C, Alfieri-Maiolo A; Pain Pain Go Away Team. The CARD ${ }^{\mathrm{TM}}$ System for improving the vaccination experience at school: results of a small-scale implementation project on program delivery. Paediatr Child Health 2019 Apr;24 Suppl 1:S54-67. DOI PubMed
14. Freedman $T$, Taddio A, Alderman L, McDowall T, deVlaming-Kot $\mathrm{C}$, McMurtry CM, MacDonald N, Alfieri-Maiolo A, Stephens D, Wong $\mathrm{H}$, Boon $\mathrm{H}$; Pain Pain Go Away Team. The CARDTM System for improving the vaccination experience at school: results of a small-scale implementation project on student symptoms. Paediatr Child Health 2019 Apr;24 Suppl 1:S42-53. DOI PubMed

15. Taddio A, Bucci LM, Logeman C, Gudzak V. Le système CARD $^{\text {TM }}$ : un outil de soins centrés sur le patient pour soulager la douleur et la peur durant les vaccinations à l'école. Ottawa (ON) : CANVax; 2020-07-21 (accédé 2020-07-30). https://canvax.ca/fr/brief/le-system e-cardtm-un-outil-de-soins-centres-sur-le-patie nt-pour-soulager-la-douleur-et-la

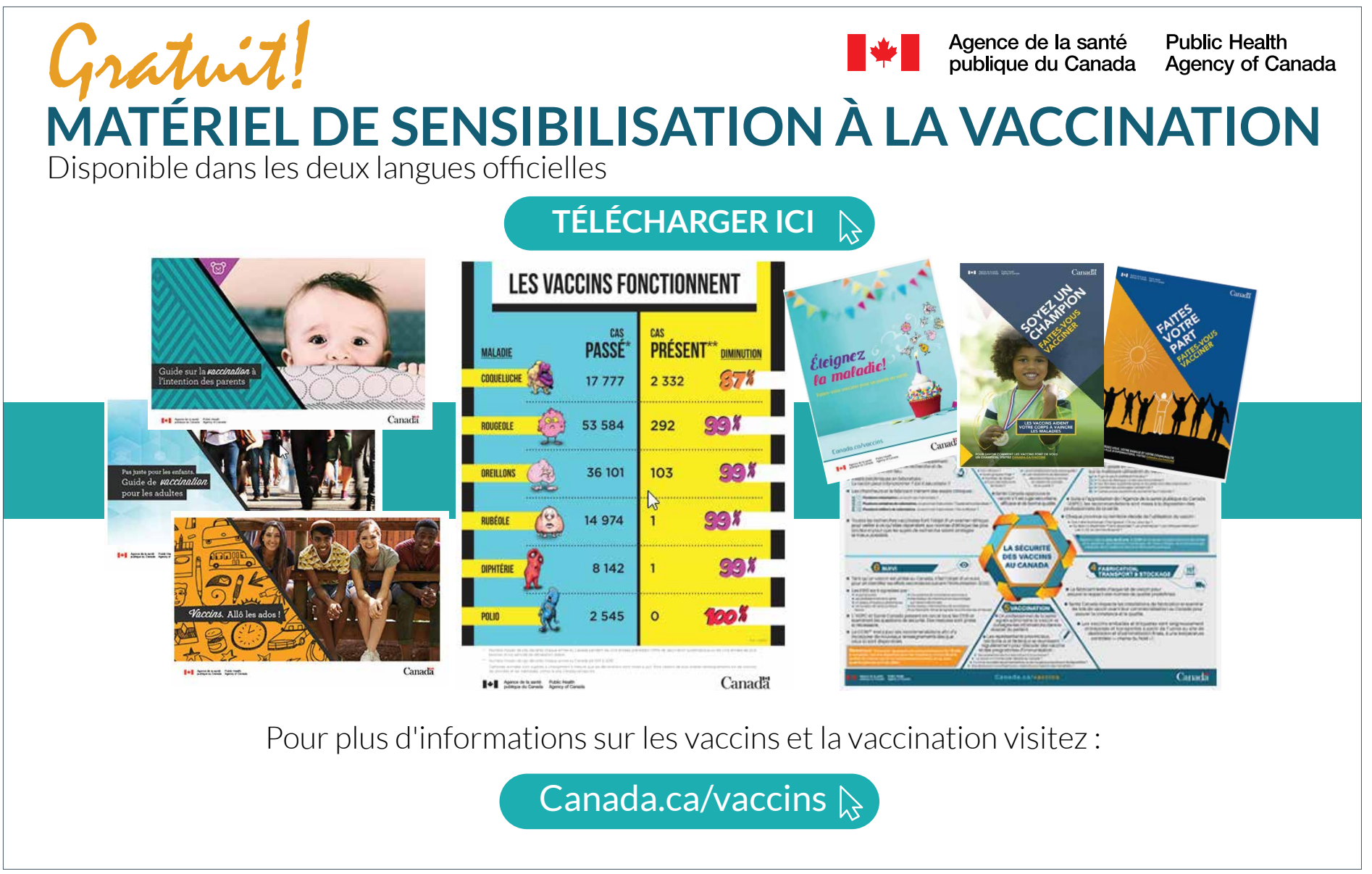

